\title{
Computational Experiments with Minimum-Distance Controlled Perturbation Methods
}

\author{
Jordi Castro ${ }^{\star}$ \\ Department of Statistics and Operations Research \\ Universitat Politècnica de Catalunya \\ Pau Gargallo 5, 08028 Barcelona, Catalonia, Spain \\ jcastro@eio.upc.es \\ http://www-eio.upc.es/ j jcastro
}

\begin{abstract}
Minimum-distance controlled perturbation is a recent family of methods for the protection of statistical tabular data. These methods are both efficient and versatile, since can deal with large tables of any structure and dimension, and in practice only need the solution of a linear or quadratic optimization problem. The purpose of this paper is to give insight into the behaviour of such methods through some computational experiments. In particular, the paper (1) illustrates the theoretical results about the low disclosure risk of the method; (2) analyzes the solutions provided by the method on a standard set of seven difficult and complex instances; and (3) shows the behaviour of a new approach obtained by the combination of two existing ones.
\end{abstract}

Keywords: statistical disclosure control, controlled perturbation methods, linear programming, quadratic programming.

\section{Introduction}

The safe dissemination of tabular data is one of the main concerns of national statistical agencies. The size and complexity of the data to be protected is continuously increasing, which results in a need for more efficient and versatile protection procedures. This work deals with minimum-distance controlled perturbation, a recent family of methods that meets the above requirements.

Currently, one of the widely used techniques in practice is cell suppression, which is known to be a NP-hard problem [15]. Although exact mixed integer linear programming procedures have been recently suggested [11], the main inconvenience of this approach is that, due to its combinatorial nature, the solution of very large instances (with possibly millions of cells) can result in impractical execution times [13]. Several heuristics have also been suggested to obtain fast approximate solutions $[1,4,7,10,15]$. Those approaches are based on the solution

\footnotetext{
* Supported by the EU IST-2000-25069 CASC project and the Spanish MCyT Project TIC2003-00997.
} 
of several network optimization subproblems. Unfortunately, although fast, they can only be applied to certain classes of tables, e.g., two and three-dimensional, and two-dimensional with hierarchies in one dimension.

To avoid the above lacks of cell suppression, alternative approaches have been introduced. One of them is the minimum-distance controlled perturbation family of methods. Given a set of tables to be protected, they find the closest ones (according to some distance measure) that, guaranteeing confidentiality, minimize the information loss. Members of that family of methods were independently suggested in [9] (the controlled table adjustment method, which uses a $L_{1}$ distance) and [3] (the quadratic minimum-distance controlled perturbation, based on $L_{2}$ ). Specialized interior-point methods [2] were used in [5] for the solution of large-scale instances. A unified framework for those methods was presented in [6], including a proof of their low disclosure risk.

This paper is organized as follows. Section 2 outlines the minimum-distance controlled perturbation framework. Section 3 illustrates the theoretical results about the disclosure risk of the method. Section 4 shows the behaviour of three particular distances on a set of seven complex instances. Finally, Section 5 reports the results obtained with an approach that combines the $L_{1}$ and $L_{2}$ distances.

\section{The Minimum-Distance Controlled Perturbation Framework}

This section only outlines the general model, and the particular formulations for the $L_{1}, L_{2}$ and $L_{\infty}$ distances. More details can be found in [9] and [6].

Any problem instance, either with one table or a number of (linked or hierarchical) tables, can be represented by the following elements:

- A set of cells $a_{i}, i=1, \ldots, n$, that satisfy some linear relations $M a=b$ ( $a$ being the vector of $a_{i}$ 's). The method will look for the closest safe values $x_{i}, i=1, \ldots, n$, according to some particular distance measure $L$, that satisfy the above constraints. The distance can be affected by any positive semidefinite diagonal metric matrix $W=\operatorname{diag}\left(w_{1}, \ldots, w_{n}\right)$.

- A lower and upper bound for each cell $i=1, \ldots, n$, respectively $\underline{a}_{i}$ and $\bar{a}_{i}$, which are considered to be known by any attacker. If no previous knowledge is assumed for cell $i, \underline{a}_{i}=0\left(\underline{a}_{i}=-\infty\right.$ if $a \geq 0$ is not required $)$ and $\bar{a}_{i}=+\infty$ can be used.

- A set $\mathcal{P}=\left\{i_{1}, i_{2}, \ldots, i_{p}\right\}$ of indices of confidential cells.

- A lower and upper protection level for each confidential cell $i \in \mathcal{P}$, respectively $l p l_{i}$ and $u p l_{i}$, such that the released values satisfy either $x_{i} \geq a_{i}+u p l_{i}$ or $x_{i} \leq a_{i}-l p l_{i}$. To add the above "or" constraint to a mathematical model we need a binary variable $y_{i}$ and two extra constraints for each confidential cell:

$$
\begin{array}{ll}
x_{i} \geq-S\left(1-y_{i}\right)+\left(a_{i}+u p l_{i}\right) y_{i} & i \in \mathcal{P}, \\
x_{i} \leq S y_{i}+\left(a_{i}-l p l_{i}\right)\left(1-y_{i}\right) & i \in \mathcal{P}, \\
y_{i} \in\{0,1\} & i \in \mathcal{P},
\end{array}
$$


$S$ in (1) being a large value. That results in a large combinatorial optimization problem which would constrain the effectiveness of the approach to small and medium sized problems. Therefore, in practice, we will assume the sense of the protection for each confidential cell (i.e., the values of the $y_{i}$ variables) is a priori fixed. This simplifying assumption permits to protect the table by the solution of a single continuous optimization problem. If the particular choice of protection senses (i.e., $y_{i}$ values) results in an infeasible problem, we can solve an alternative one by relaxing the constraints $M a=b$ with a large penalization for possible perturbations in the right-hand-side (see Section 5 for details).

The general minimum-distance controlled perturbation method, using some $L$ distance, can be formulated as the following optimization problem:

$$
\begin{aligned}
\min _{x} & \|x-a\|_{L} \\
\text { subject to } & M x=b \\
& \underline{a}_{i} \leq x_{i} \leq \bar{a}_{i} \quad i=1, \ldots, n \\
& x_{i} \leq a_{i}-l p l_{i} \text { or } x_{i} \geq a_{i}+u p l_{i} \quad i \in \mathcal{P} .
\end{aligned}
$$

The general problem (2) can also be formulated in terms of deviations or perturbations from the current cell values. Defining $z_{i}=x_{i}-a_{i}, \quad i=1, \ldots, n$, (2) can be transformed to

$$
\begin{aligned}
\min _{z} & \|z\|_{L} \\
\text { subject to } & M z=0 \\
& \underline{z}_{i} \leq z_{i} \leq \bar{z}_{i} \quad i=1, \ldots, n \\
& z_{i} \leq-l p l_{i} \text { or } z_{i} \geq u p l_{i} \quad i \in \mathcal{P} .
\end{aligned}
$$

where $z \in \mathbb{R}^{\mathrm{n}}$ is the vector of deviations, $\underline{z}_{i}=\underline{a}_{i}-a_{i} \leq 0$ and $\bar{z}_{i}=\bar{a}_{i}-a_{i} \geq 0$. A benefit of (3) is that it can be solved without releasing the confidential data vector $a$.

Using the $L_{1}$ distance, and after some manipulation, (3) can be written as

$$
\begin{aligned}
\min _{z^{+}, z^{-}} & \sum_{i=1}^{n} w_{i}\left(z_{i}^{+}+z_{i}^{-}\right) \\
\text {subject to } & M\left(z^{+}-z^{-}\right)=0 \\
& 0 \leq z_{i}^{+} \leq \bar{z}_{i} \quad i=1, \ldots, n \\
& 0 \leq z_{i}^{-} \leq-\underline{z}_{i} \quad i=1, \ldots, n \\
& \left\{\begin{array}{l}
z_{i}^{+} \geq u p l_{i} \\
z_{i}^{-}=0
\end{array}\right\} \quad \text { or } \quad\left\{\begin{array}{l}
z_{i}^{-} \geq l p l_{i} \\
z_{i}^{+}=0
\end{array}\right\} \quad i \in \mathcal{P},
\end{aligned}
$$

$z^{+}$and $z^{-}$being the vector of positive and negative deviations in absolute value.

For $L_{2},(3)$ is

$$
\begin{aligned}
\min _{z} & \sum_{i=1}^{n} w_{i} z_{i}^{2} \\
\text { subject to } & M z=0 \\
& \underline{z}_{i} \leq z_{i} \leq \bar{z}_{i} \quad i=1, \ldots, n \\
& z_{i} \leq-l p l_{i} \text { or } z_{i} \geq \text { upl } i \quad i \in \mathcal{P} .
\end{aligned}
$$


Finally, for $L_{\infty}$, the general model (3) can be formulated as

$$
\begin{aligned}
& \min _{z^{+}, z^{-}, z_{\in \mathcal{P}}, z_{\notin \mathcal{P}}} z_{\in \mathcal{P}}+z_{\notin \mathcal{P}} \\
& \text { subject to } M\left(z^{+}-z^{-}\right)=0 \\
& 0 \leq z_{i}^{+} \leq \bar{z}_{i} \quad i=1, \ldots, n \\
& 0 \leq z_{i}^{-} \leq-\underline{z}_{i} \quad i=1, \ldots, n \\
& \left\{\begin{array}{l}
z_{i}^{+} \geq u p l_{i} \\
z_{i}^{-}=0
\end{array}\right\} \quad \text { or } \quad\left\{\begin{array}{l}
z_{i}^{-} \geq l p l_{i} \\
z_{i}^{+}=0
\end{array}\right\} \quad i \in \mathcal{P} \\
& z_{\in \mathcal{P}} \geq w_{i}\left(z_{i}^{+}+z_{i}^{-}\right) \quad i \in \mathcal{P} \\
& z_{\notin \mathcal{P}} \geq w_{i}\left(z_{i}^{+}+z_{i}^{-}\right) \quad i \notin \mathcal{P},
\end{aligned}
$$

$z_{\in \mathcal{P}}$ and $z_{\notin \mathcal{P}}$ being extra variables that store the maximum deviation for, respectively, the sensitive and nonsensitive cells.

An appropriate choice for the weights in $(4-6)$ is $w_{i}=1 / a_{i}$, making the deviations relative to the cell value. These weights will be used in the computational results of the paper. (4) is a fixed version of the controlled tabular adjustment suggested in [9]. $L_{2}$ provides the smallest optimization problem, although it is quadratic. $L_{1}$ and $L_{\infty}$ provide linear problems, with a larger number of variables and constraints. Effective approaches for the solution of (4-6) were discussed in [6].

\section{Illustrating the Disclosure Risk of the Method}

The theoretical results about the disclosure risk of the method were presented in [6]. This section summarizes them, and illustrates the low disclosure risk of the method through an example.

To retrieve the original table, the attacker should compute the deviations applied by solving the optimization problem (3). In practice the only term known by the attacker is the $M$ matrix provided by the table structure. However, assume the attacker has partial information, $u p l_{i}, i \in \mathcal{P}$, being the only unknown terms (without loss of generality we consider all the protection senses were "upper"). The problem to be solved to disclose the deviations is then

$$
\begin{aligned}
\min _{z^{\prime}} & \left\|z^{\prime}\right\|_{L} \\
\text { subject to } & M z^{\prime}=0 \\
& z_{i}^{\prime} \geq u p l_{i}+e_{i}, \quad i \in \mathcal{P},
\end{aligned}
$$

$u p l_{i}+e_{i}$ being the approximate values used by the attacker to obtain the approximate deviations $z^{\prime}$. The protection of the table thus depends on how sensitive the solution $z^{\prime *}$ is to possible small $e_{i}$ values. This relation is explained by the next proposition [6]:

Proposition 1. If $z^{\prime *}(e) \in \mathbb{R}^{\mathrm{n}}$ is the solution of (7) for a particular vector of $e=\left(e_{1}, \ldots, e_{|\mathcal{P}|}\right)$ values, and $\mu \in \mathbb{R}^{|\mathcal{P}|}$ is the Lagrange multipliers vector of the bounds of $z^{\prime}$ in (7) for $e=0$ (i.e., the multipliers obtained when protecting the table), then

$$
\left.\nabla_{e}\left\|z^{\prime *}(e)\right\|_{L}\right|_{e=0}=\mu \text {. }
$$




\begin{tabular}{|cccc|c|}
\hline $\mathbf{1 0}_{(\mathbf{3})}$ & 15 & 11 & 9 & 45 \\
8 & 10 & $\mathbf{1 2}_{(\mathbf{4})}$ & 15 & 45 \\
10 & 12 & $\mathbf{1 1}_{(\mathbf{2})}$ & $\mathbf{1 3}_{(\mathbf{5})}$ & 46 \\
\hline 28 & 37 & 34 & 37 & 136 \\
\hline
\end{tabular}

(a)

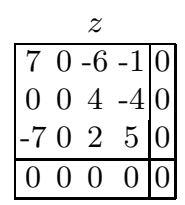

(b)

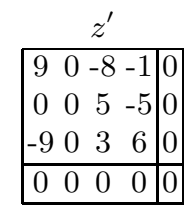

(c)

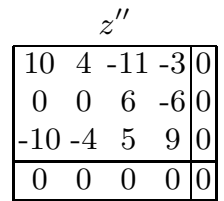

(d)

Fig. 1. Example of sensitivity of the method to changes in the protection levels. (a) Original data $a$ to be protected. Sensitive cells are in boldface, and upper protection levels are given in brackets. (b) Optimal deviations $z$ computed with the $L_{1}$ distance, weights $w_{i}=1$, and inactive bounds $\underline{a_{i}}=0$ and $\overline{a_{i}}=\infty$ for all the internal cells. Marginal cells were fixed. The Lagrange multipliers of the bounds $z_{i} \geq u p l_{i}$ for the sensitive cells are $\mu_{11}=0, \mu_{23}=2, \mu_{33}=4$ and $\mu_{34}=4$. The objective function - the sum of deviations in absolute value - is 36. (c) and (d) Deviations $z^{\prime}$ and $z^{\prime \prime}$ computed by the attacker using approximate protection levels with errors $e_{11}=e_{23}=e_{33}=e_{34}=1$, and $e_{11}=1, e_{23}=2, e_{33}=3, e_{34}=4$, respectively. The objective functions are respectively 46 and 68 which satisfy (9).

Moreover, for, respectively, the $L_{1}$ and $L_{\infty}$ distances, problem (7) is linear, and, for small enough vectors $e=\left(e_{1}, \ldots, e_{|\mathcal{P}|}\right),(8)$ can be recast as

$$
\left\|z^{\prime *}(e)\right\|_{L}-\left\|z^{*}\right\|_{L}=\sum_{i \in \mathcal{P}} \mu_{i} e_{i}
$$

$z^{*}$ being the deviations used to protect the table.

To illustrate the above result, consider the example of Figure 1. Table (a) shows the original data to be protected. Sensitive cells appear in boldface, and their upper protection levels $u p l_{i}$ are given in brackets. Using the $L_{1}$ distance, weights $w_{i}=1$, and bounds $a_{i}=0$ and $\overline{a_{i}}=\infty$ for all the internal cells, the optimal deviations computed are shown in Table (b). The objective function value is $\|z\|_{L_{1}}=\sum_{i=1}^{n}\left|z_{i}\right|=36$. The Lagrange multipliers of the constraints $z_{i} \geq u p l_{i}$ for the sensitive cells are $\mu_{11}=0, \mu_{23}=2, \mu_{33}=4$ and $\mu_{34}=4$. Since bounds $\underline{a_{i}}=0$ are inactive in the solution, the attacker can use (7) to disclose the deviations of Table (b). If, for instance, the attacker can adjust all the original $u p l_{i}$ protection levels, but for cell $a_{11}$, (in this case, if $e_{11} \leq 4$, $e_{23}=e_{33}=e_{34}=0$ ), from (9) and since $\mu_{11}=0$, a solution with the same objective function (and possibly with the same deviations) that for Table (b) (i.e., 36) will be obtained. However, if all the protection levels are adjusted with errors, a different solution will be computed. For instance, if problem (7) is solved with $e_{11}=e_{23}=e_{33}=e_{34}=1$, the deviations $z^{\prime}$ obtained are those of Table (c). The objective function (i.e., sum of deviations) is 46, which satisfies (9): $46-36=1 \mu_{11}+1 \mu_{23}+1 \mu_{33}+1 \mu_{34}$. If problem (7) is solved with slightly larger values $e_{11}=1, e_{23}=2, e_{33}=3, e_{34}=4$, the deviations $z^{\prime \prime}$ obtained are shown in Table (c). Again, the objective function, 68, satisfies: $68-36=$ $1 \mu_{11}+2 \mu_{23}+3 \mu_{33}+4 \mu_{34}$.

Note that $\left\|\left(\mu_{i: i \in \mathcal{P}}\right)\right\|$ (the norm of the Lagrange multipliers of constraints $z_{i} \geq u p l_{i}$ for the sensitive cells) can be used as an indicator of the protection of 


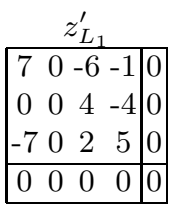

(a)

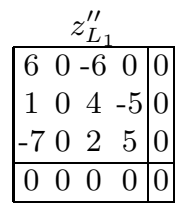

(b)

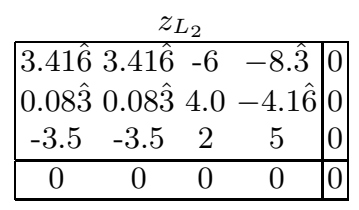

(c)

Fig. 2. Example of alternative solutions with complete information by the attacker. The original data $a$ to be protected are those of Table (a) of Figure 1. Sensitive cells are in boldface, and upper protection levels are given in brackets. (a) and (b) Alternative solutions $z_{L_{1}}^{\prime}$ and $z_{L_{1}}^{\prime \prime}$, computed with two different linear programming solvers, using the $L_{1}$ distance, weights $w_{i}=1$, and bounds $\underline{a_{i}}=0$ and $\overline{a_{i}}=\infty$ for all the internal cells. Marginal cells were fixed. The objective function - the sum of deviations in absolute value - of both solutions is 36. (c) Unique solution $z_{L_{2}}$ for the $L_{2}$ distance, again with weights $w_{i}=1$, and bounds $\underline{a_{i}}=0$ and $\overline{a_{i}}=\infty$ for all the internal cells. The 2-norm of the deviations vector is $12 . \overline{12}$.

the table. In theory, the larger this value, the more difficult is for an attacker to retrieve the original data. Real tables, with a large number of sensitive cells, often will have a high $\left\|\left(\mu_{i: i \in \mathcal{P}}\right)\right\|$ value, and thus confidential.

In some cases, even if the attacker has complete information, the right perturbations can not be disclosed [6]:

Proposition 2. Assume the attacker knows all the terms of problem (7). If the $L_{2}$ distance is used, the solution of that problem will provide the deviations used to protect the table. However, for $L_{1}$ or $L_{\infty}$, the attacker can obtain alternative deviations.

For instance, Tables (a) and (b) of Figure 2 show two alternative solutions with the $L_{1}$ distance for the data of Table (a) of Figure 1. They were obtained with two different implementations of the simplex algorithm, using weights $w_{i}=$ 1 , and bounds $\underline{a}_{i}=0$ and $\overline{a_{i}}=\infty$ for all the internal cells. Marginal cells were fixed. The sum of deviations is 36 in both solutions. Table (c) of Figure 2 shows, for the same data, the unique solution for the $L_{2}$ distance. Since $L_{2}$ involves a quadratic function, the solution attempts to distribute the deviations among all the cells, obtaining a non-integer solution (valid for magnitude tables). Proposition 2 means that $L_{1}$ and $L_{\infty}$ are a bit safer when the attacker knows all the terms of $(7)$, which in practice is equivalent to that the attacker knows the original data (thus, very unlikely). Therefore, in practice, it can be concluded that the three distances have the same low disclosure risk.

\section{Computational Comparison}

For the computational comparison of models (4-6) (i.e., $L_{1}, L_{2}$ and $L \infty$ ) we used the seven most complex instances of CSPLIB. CSPLIB is the unique currently available set of instances for tabular data protection [11]. It can be freely 
Table 1. Properties of the seven complex instances.

\begin{tabular}{lllllll}
\hline Name & Dimensions & Size & $n$ & $|\mathcal{P}|$ & $m$ & N.coef \\
\hline bts4 & 4D, hierarchical & $54,54,4,4$ & 36570 & 2260 & 36310 & 136912 \\
hier13 & 3D, hierarchical & $13,13,13$ & 2020 & 112 & 3313 & 11929 \\
hier16 & 3D, hierarchical & $16,16,16$ & 3564 & 224 & 5484 & 19996 \\
nine12 & 9D, linked & $10,6,6,6,6,6,6,6,6$ & 10399 & 1178 & 11362 & 52624 \\
nine5d & 9D, linked & $4,29,3,4,5,6,5,4,5$ & 10733 & 1661 & 17295 & 58135 \\
ninenew & 9D, linked & $10,6,6,6,6,6,6,6,6$ & 6546 & 858 & 7340 & 32920 \\
two5in6 & 6D, linked & $6,4,16,4,4,4$ & 5681 & 720 & 9629 & 34310 \\
\hline
\end{tabular}

obtained from http://webpages.ull.es/users/casc/\#CSPlib: . These seven instances were also the choice in [8] and are challenging for other approaches, as cell suppression. As shown below, they can be solved in few seconds with the minimum-distance approach. Table 1 provides their main features: identifier (column "Name"), number of dimensions and structure - linked or hierarchical - (column "Dimensions"), size for each dimension (column "Size"), number of total cells and sensitive cells (columns " $n$ " and "|P्P|", respectively), number of constraints (column " $m$ "), and number of coefficient of the $M$ matrix (column "N.coef"). The structure and size information was obtained from [8].

Problems (4-6) were implemented using the AMPL modelling language [12] and CPLEX 8.0 [14]. All runs were carried on a notebook with a $1.8 \mathrm{GHz}$ processor and $512 \mathrm{Mb}$ of RAM. For $L_{2}$ we used the primal-dual interior-point algorithm [16], which can be considered the most efficient choice. $L_{1}$ and $L_{\infty}$ were solved with the two best linear programming algorithms: the simplex method and the primal-dual interior-point method. Although the optimal objective function is the same, both algorithms can provide different solutions. In this work we used those of the simplex method, which, in practice, provided better deviations.

For each of the three distances, Table 3 of Appendix A show the following information. Row "CPU" gives the CPU time in seconds for each algorithm. Rows "Abs. dev." provide the mean (columns "mean"), standard deviation (columns "std") and maximum (columns "max.") of the absolute deviations (i.e., $\left|z_{i}\right|$ ), for all the cells (row "all"), for the sensitive cells (row " $\in \mathcal{P}$ "), and for the nonsensitive cells (row " $\notin \mathcal{P}$ "). A similar information is provided for the percentage absolute deviations (i.e., 100| $z_{i} \mid / a_{i}$ ) in rows "Perc. dev.". Finally, rows "2-norm" report the 2 -norm of the deviations (i.e., $\|z\|_{2}$ ), again for sensitive, nonsensitive, and all the cells.

Looking at Table 3 we see that most of the optimization problems were solved until optimality in few seconds on a standard personal computer. $L_{\infty}$ provides the slowest executions, due to the large number of constraints considered in (6). $L_{2}$, solved through a quadratic interior-point solver, was always the most efficient choice (except for the smallest instance hier13). In most instances the solution time of the $L_{2}$ was about half the time of the second fastest option. This is because, first, the complexity of solving a quadratic separable optimization problem is the same that for a linear one, if we use an interior-point algorithm; and second, problem (4) involves the double of variables that (5). The solu- 
tion times obtained with the interior-point algorithm, for the three objectives, can even be improved using specialized solvers that exploit the tables structure $[2,5]$.

For the absolute deviations, $L_{2}$ provides the lowest means and, mainly, the lowest standard deviations. Such lowest standard deviations are not surprising, since $L_{2}$, due to its quadratic nature, attempts to evenly distribute the required deviations among all the cells. As for the other two distances, $L_{\infty}$ provided better absolute deviations than $L_{1}$, but for instances hier13 and hier16. That was, a priori, an unexpected result, since only two cells appear in the objective function of (6), whereas all the perturbations are considered in (4). The distribution of the absolute deviations (not reported in the tables) showed that $L_{1}$ provided the greater number of cells with small deviations.

For the percentage deviations, $L_{1}$ must clearly provide the best mean values, since its objective function is exactly the sum of percentage absolute deviations. However, $L_{2}$ provides similar mean percentage deviations, and, for most instances, with slightly better standard deviations. $L_{\infty}$ provided worser means and standard deviations, but, as a consequence of its objective function, the lowest maximum values.

Finally, the lowest 2-norms of the deviations vector are provided in all the instances by $L_{2}$. This is a consequence of $L_{2}$ being the only quadratic objective of the three tested. Except for instance hier13, $L_{\infty}$ always provides deviations with better 2-norms than $L_{1}$.

From the above comments, we can conclude that $L_{1}$ provides the best results when a first-order comparison measure, as the mean percentage deviation, is considered. However, when a second-order measure is used, as the 2-norm of the deviations or the standard deviation of the percentage deviations, $L_{2}$ seems to be the best choice. The above is an immediate result of the objective functions (linear or quadratic) of the respective optimization problems. That suggests that a method combining $L_{1}$ and $L_{2}$ could provide fairly good values for the first and second-order comparison measures. This alternative is exploited in next section.

\section{Combining the $L_{1}$ and $L_{2}$ Distances}

The optimization problem that results from the combination of the $L_{1}$ and $L_{2}$ distances can be written in a general form as

$$
\begin{aligned}
\min _{z^{+}, z^{-}} & \omega_{1} \sum_{i=1}^{n} w_{1, i}\left(z_{i}^{+}+z_{i}^{-}\right)+\omega_{2} \sum_{i \in \mathcal{S}} w_{2, i}\left(z_{i}^{+}+z_{i}^{-}\right)^{2} \\
\text { subject to } & M\left(z^{+}-z^{-}\right)=0 \\
& 0 \leq z_{i}^{+} \leq \bar{z}_{i} \quad i=1, \ldots, n \\
& 0 \leq z_{i}^{-} \leq-\underline{z}_{i} \quad i=1, \ldots, n \\
& \left\{\begin{array}{l}
z_{i}^{+} \geq u p l_{i} \\
z_{i}^{-}=0
\end{array}\right\} \quad \text { or } \quad\left\{\begin{array}{l}
z_{i}^{-} \geq l p l_{i} \\
z_{i}^{+}=0
\end{array}\right\} \quad i \in \mathcal{P},
\end{aligned}
$$

$z^{+}$and $z^{-}$being the vector of positive and negative deviations in absolute value, $\omega_{1}$ and $\omega_{2}$ weights for the overall contribution to the objective function of re- 


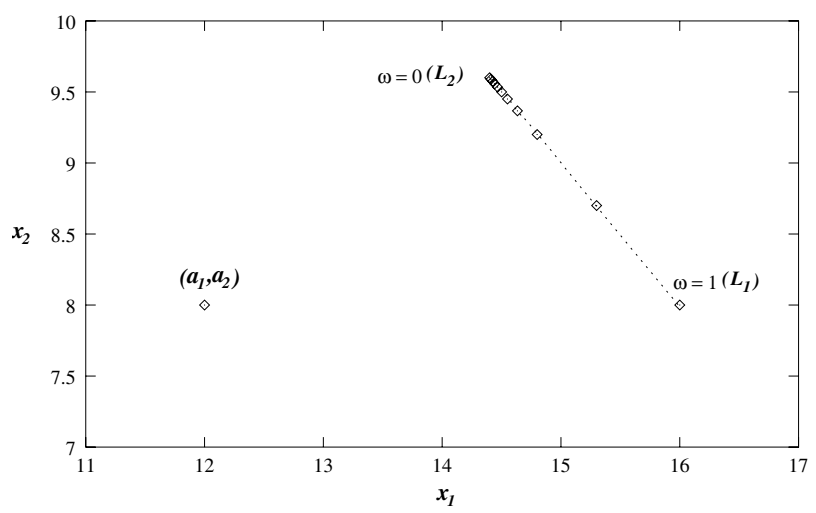

Fig. 3. Solutions of the $L_{1}$ and $L_{2}$ distances for the one dimensional table $a_{1}+a_{2}=$ $a_{3}$, imposing a perturbation $z_{3} \geq 4$ for the marginal cell. Point $\left(a_{1}, a_{2}\right)=(12,8)$ corresponds to the original internal cell values. The other eleven points are the solutions obtained with the objective function of (4) using $\omega_{1}=\omega$ and $\omega_{2}=1-\omega$, for $\omega=$ $0,0.1,0.2, \ldots, 0.9,1$, which combines the $L_{1}$ and $L_{2}$ distances through the weight factor $\omega$. The $L_{2}$ solution (computed with $\left.\omega=0\right)$ is closer to $\left(a_{1}, a_{2}\right)$, but the $L_{1}$ point $(\omega=1)$ preserves the value of $a_{2}$.

spectively the linear and quadratic terms, $\mathcal{S}$ a subset of cells affected by $L_{2}$, and $w_{1, i}$ and $w_{2, i}$ cell weights for respectively $L_{1}$ and $L_{2}$. This formulation is general enough to accommodate to several situations. For instance, it provides an always feasible problem if we apply the $L_{1}$ and $L_{2}$ terms to respectively the internal and marginal cells (i.e., $\mathcal{S}$ is the set of marginal cells), with a large penalization for changes in marginal values (i.e., $w_{2, i} \gg 0$ ).

Before presenting results for the seven instances of Section 4, we first illustrate the behaviour of (10) on the small example of Figure 3. The table considered is $a_{1}+a_{2}=a_{3}$, with $a_{1}=12$ and $a_{2}=8$. We imposed $z_{1}+z_{2}=z_{3}$ and $z_{3} \geq 4$, i.e., an upper protection level of 4 is forced for the marginal sensitive cell. We set $\mathcal{S}=\{1,2,3\}$ (i.e., the three cells appear in the quadratic term of the objective function), and $\omega_{1}=\omega, \omega_{2}=1-\omega, \omega \in[0,1]$ being a predefined parameter. For $\omega=1$ and $\omega=0$ the combined objective of (10) corresponds to the $L_{1}$ and $L_{2}$ distances, respectively. Using $w_{1, i}=1 / a_{i}$ the optimal solution obtained with $L_{1}$ is $z_{1}=4, z_{2}=0$ and $z_{3}=4$. With the same weights $w_{2, i}=$ $1 / a_{i}$, the optimal solution provided by $L_{2}$ is $z_{1}=2.4, z_{2}=1.6$ and $z_{3}=4$. If integer values were required, the $z_{1}$ and $z_{2}$ values could be rounded through some heuristic postprocess (in that case the most reasonable choice would be $z_{1}=2$ and $z_{2}=2$ ). Figure 3 shows the perturbed internal cell values obtained for $\omega=0,0.1, \ldots, 0.9,1$, and the original ones $\left(a_{1}, a_{2}\right)$. Clearly, the $L_{2}$ point is closer to $(12,8)$, but the $L_{1}$ solution preserves the value of cell $a_{2}$. This is consistent with the results of Section 4 . The combined $L_{1-2}$ objective provides solutions on a curve joining the $L_{1}$ and $L_{2}$ points. Because of the larger costs of the quadratic term, the optimal solution was only far enough from the $L_{2}$ point for $\omega=0.8$ and $\omega=0.9$. 
Table 2. Results for the seven complex instances, for $L_{1}, L_{2}$ and $L_{1-2}$.

\begin{tabular}{|c|c|c|c|c|c|c|c|c|c|}
\hline \multirow[b]{2}{*}{ name } & \multicolumn{3}{|c|}{$L_{1}$} & \multicolumn{3}{|c|}{$L_{2}$} & \multicolumn{3}{|c|}{$L_{1-2}$} \\
\hline & $\mathrm{CPU}$ & $\overline{7 \mathrm{Dev}}$ & 2-norm & $\mathrm{CPU}$ & $6 \overline{\mathrm{Dev}}$ & -norm & $\mathrm{CPU}$ & $\overline{\mathrm{Dev} .}$ & 2-norm \\
\hline bts4 & 16.5 & 0.74 & 18243 & 11.5 & 0.83 & 7912 & 45.0 & 0.76 & 10217 \\
\hline hier13 & 3.3 & 0.81 & 2609 & 3.8 & 0.87 & 2149 & 7.1 & 0.82 & 2306 \\
\hline hier16 & 19.9 & 0.83 & 3203 & 17.1 & 0.90 & 2706 & 31.0 & 0.84 & 2845 \\
\hline nine12 & 382.1 & 1.35 & 5840 & 18.3 & 1.53 & 4878 & 43.7 & 1.38 & 5234 \\
\hline nine $5 d$ & 126.7 & 1.67 & 8316 & 20.4 & 1.90 & 5468 & 30.9 & 1.72 & 5845 \\
\hline ninenew & 27.0 & 1.55 & 5448 & 11.1 & 1.76 & 4444 & 26.3 & 1.56 & 4731 \\
\hline two5in6 & 13.6 & 1.46 & 4917 & 9 & 1.65 & 3749 & 16.8 & 1.50 & 4045 \\
\hline
\end{tabular}

In the computational results of this section we used $\mathcal{S}=\{1, \ldots, n\}$ (i.e., all the cells are involved in the quadratic term) and $w_{1, i}=w_{2, i}=1 / a_{i}, i=1, \ldots, n$. According to the previous small example, we also set $\omega_{1}=0.99$ and $\omega_{2}=0.01$. Table 2 shows the results obtained with $L_{1}, L_{2}$ and the combined $L_{1-2}$ objective. For each distance, the execution time (columns "CPU"), average percentage deviation for all the cells (columns "\%Dev."), and 2-norm of the deviations vector (columns "2-norm") are provided. Executions were carried on the same hardware and with the same software (i.e., AMPL+CPLEX 8.0) than in Section 4. The results reported for $L_{1}$ were obtained with the simplex method, while the quadratic interior-point algorithm was used for $L_{2}$ and $L_{1-2}$. Looking at Table 2 we see the combined $L_{1-2}$ distance provides average percentage deviations close to those of $L_{1}$, while the 2-norm has been significantly reduced. As expected, the combined $L_{1-2}$ distance inherited the good properties of $L_{1}$ and $L_{2}$.

\section{Conclusions}

As shown by the computational experiments of this work, the minimum-distance approach is efficient, versatile and safe. The three methods tested, for $L_{1}, L_{2}$ and $L_{\infty}$, provided different patterns of deviations, each of them with a clear behaviour. As done in the paper with $L_{1}$ and $L_{2}$, it is possible to combine them in a new approach with the good features of the original methods.

One of the fields of research to be be explored deals with the optimization solvers. In a static environment, the final goal might be the protection, in a single run, of all the tables derived from the same microdata. The resulting problem is huge. In a dynamic environment, the goal would be the online protection of particular tables (e.g., obtained from end-user queries from a data-warehouse). Speed is instrumental in that case. In both situations, we may need highlyefficient implementations of the optimization methods used in this work, which exploit the problem structure. Some steps have already been done in this direction for large (i.e., one million cells) three-dimensional tables and $L_{2}$ [5], where a specialized implementation was two orders of magnitude faster than the CPLEX 8.0 solver. Extending those achievements to general tables is part of the future work to be done. 


\section{References}

1. Carvalho, F.D., Dellaert, N.P., Osório, M.D.: Statistical disclosure in twodimensional tables: general tables. J. Am. Stat. Assoc. 89 (1994) 1547-1557

2. Castro, J.: A specialized interior-point algorithm for multicommodity network flows. SIAM J. on Opt. 10 (2000) 852-877

3. Castro, J.: Internal communication to partners of the European Union IST-200025069 CASC project (2002).

4. Castro, J.: Network flows heuristics for complementary cell suppression: an empirical evaluation and extensions. Lect. Notes in Comp. Sci. 2316 (2002) 59-73. Volume Inference Control in Statistical Databases, ed. J. Domingo-Ferrer. Springer.

5. Castro, J.: Quadratic interior-point methods in statistical disclosure control, Computational Management Science, in press (2004). Previously appeared as Research Report DR 2003/10, Dept. of Statistics and Operations Research, Universitat Politècnica de Catalunya (2003). Available from the author webpage.

6. Castro, J.: Minimum-distance controlled perturbation methods for large-scale tabular data protection. European Journal of Operational Research, accepted subject to revision (2003). An extended version previously appeared as Research Report DR 2003/14, Dept. of Statistics and Operations Research, Universitat Politècnica de Catalunya (2003). Available from the author webpage.

7. Cox, L.H.: Network models for complementary cell suppression. J. Am. Stat. Assoc. 90 (1995) 1453-1462

8. Dandekar, R.A.: Cost effective implementation of synthetic tabulation (a.k.a. controlled tabular adjustments) in legacy and new statistical data publication systems. Joint ECE/Eurostat Work Session on Statistical Data Confidentiality, Luxembourg, (2003). Available from http://www . unece.org/stats/documents/2003.04.confidentiality.htm.

9. Dandekar, R.A., Cox, L.H.: Synthetic tabular data: an alternative to complementary cell suppression. Manuscript, Energy Information Administration, U.S. Department of Energy (2002). Available from the first author on request (Ramesh.Dandekar@eia.doe.gov).

10. Dellaert, N.P., Luijten, W.A.: Statistical disclosure in general three-dimensional tables. Statistica Neerlandica 53 (1999) 197-221

11. Fischetti, M., Salazar, J.J.: Models and algorithms for optimizing cell suppression in tabular data with linear constraints. J. Am. Stat. Assoc. 95 (2000) 916-928

12. Fourer, R, Gay, D.M., Kernighan, B.W.: AMPL: A Modeling Language for Mathematical Programming. Duxbury Press (1993)

13. Giessing, S.: New tools for cell-suppression in $\tau$-Argus: one piece of the CASC project work draft. Joint ECE/Eurostat Work Session on Statistical Data Confidentiality, Skopje (2001).

14. ILOG CPLEX: ILOG CPLEX 8.0 Reference Manual Library. ILOG (2002)

15. Kelly, J.P., Golden, B.L, Assad, A.A.: Cell Suppression: disclosure protection for sensitive tabular data. Networks 22 (1992) 28-55

16. Wright, S.J.: Primal-Dual Interior-Point Methods. SIAM (1997). 


\section{Appendix}

\section{A Tables with Results for the Seven Instances}

Table 3. Results for the seven complex instances, for $L_{1}, L_{2}$ and $L_{\infty}$.

Results for the bts4 instance

\begin{tabular}{|c|c|c|c|c|c|c|c|c|c|c|c|c|}
\hline \multirow{5}{*}{$\begin{array}{l}\text { CPU } \\
\text { Abs. } \\
\text { dev. }\end{array}$} & \multicolumn{4}{|c|}{$L_{1}$} & \multicolumn{4}{|c|}{$L_{2}$} & \multicolumn{4}{|c|}{$L_{\infty}$} \\
\hline & \multicolumn{2}{|c|}{$\begin{array}{c}\text { Simplex } \\
16.46 \\
\end{array}$} & \multicolumn{2}{|c|}{$\begin{array}{c}\text { Int. Point } \\
39.7\end{array}$} & \multicolumn{4}{|c|}{$\begin{array}{c}\text { Int. Point } \\
11.45 \\
\end{array}$} & \multicolumn{2}{|c|}{$\begin{array}{l}\text { Simplex } \\
1594.69\end{array}$} & \multicolumn{2}{|c|}{$\begin{array}{l}\text { Int. Point } \\
207.02\end{array}$} \\
\hline & & mean & std & $\max$. & & lean & std & & & mean & std & $\max$. \\
\hline & all & 33.9 & 89.2 & & all & 24.9 & 33.0 & & all & 30.1 & 49.0 & 947.8 \\
\hline & $\in \mathcal{P}$ & 56.0 & 32.2 & 155.0 & $\in \mathcal{P}$ & 56.0 & 32.2 & 155.0 & $\in \mathcal{P}$ & 57.0 & 32.6 & 168.5 \\
\hline \multirow{5}{*}{$\begin{array}{l}\text { Perc. } \\
\text { dev. }\end{array}$} & $\notin \mathcal{P}$ & 32.4 & 91.5 & 4483.0 & $\notin \mathcal{P}$ & 22.9 & 32.0 & 795.9 & $\notin \mathcal{P}$ & 28.4 & 49.4 & 947.8 \\
\hline & & mean & std & $\mathrm{ma}$ & & mean & std & & & mean & std & $\max$ \\
\hline & all & .74 & 1.97 & & all & 0.84 & 1.95 & & all & 1.10 & 2.36 & 11.11 \\
\hline & $\in \mathcal{P}$ & 7.27 & 2.60 & 11 . & $\in \mathcal{P}$ & 7.27 & 2.59 & 11 & $\in \mathcal{P}$ & 7.46 & 2.61 & 11.11 \\
\hline & $\notin \mathcal{P}$ & 0.31 & 0.83 & 11.03 & $\notin \mathcal{P}$ & 0.42 & 0.84 & 20.23 & $\notin \mathcal{P}$ & 0.68 & 1.63 & 11.0 \\
\hline \multirow{3}{*}{ norm } & \multicolumn{2}{|l|}{ all } & \multicolumn{2}{|c|}{18243.0} & \multicolumn{2}{|l|}{ all } & \multicolumn{2}{|l|}{7912.0} & all & \multirow{2}{*}{\multicolumn{3}{|c|}{10997.2}} \\
\hline & \multicolumn{2}{|l|}{$\in \mathcal{P}$} & \multicolumn{2}{|c|}{3072.3} & \multirow{2}{*}{\multicolumn{2}{|c|}{$\begin{array}{l}\in \mathcal{P} \\
\notin \mathcal{P}\end{array}$}} & \multicolumn{2}{|l|}{3070.3} & $\in \mathcal{P}$ & & & \\
\hline & \multicolumn{2}{|l|}{$\notin \mathcal{P}$} & \multicolumn{2}{|c|}{17982.4} & & & 7292.0 & & $\notin \mathcal{P}$ & & 10545. & \\
\hline
\end{tabular}

Results for the hier13 instance

\begin{tabular}{|c|c|c|c|c|c|c|c|c|c|c|c|c|}
\hline \multirow[b]{2}{*}{$\mathrm{CPU}$} & \multicolumn{4}{|c|}{$L_{1}$} & \multicolumn{4}{|c|}{$L_{2}$} & \multicolumn{4}{|c|}{$L_{\infty}$} \\
\hline & \multicolumn{2}{|c|}{$\begin{array}{c}\text { Simplex } \\
3.25\end{array}$} & \multicolumn{2}{|c|}{$\begin{array}{c}\text { Int. Point } \\
6.86\end{array}$} & \multicolumn{4}{|c|}{$\begin{array}{c}\text { Int. Point } \\
3.83 \\
\end{array}$} & \multicolumn{2}{|c|}{$\begin{array}{c}\text { Simplex } \\
5.85\end{array}$} & \multicolumn{2}{|c|}{$\begin{array}{c}\text { Int. Point } \\
35.23\end{array}$} \\
\hline \multirow{3}{*}{$\begin{array}{l}\text { Abs. } \\
\text { dev. }\end{array}$} & all & $\begin{array}{l}\text { mean } \\
37.8\end{array}$ & $\begin{array}{l}\text { std } \\
44.1\end{array}$ & $\begin{array}{l}\max . \\
344.0\end{array}$ & all & $\begin{array}{l}\text { mean } \\
33.9\end{array}$ & $\begin{array}{l}\text { std } \\
33.7\end{array}$ & $\begin{array}{l}\max . \\
313.4\end{array}$ & all & $\begin{array}{l}\text { mean } \\
52.0\end{array}$ & $\begin{array}{l}\text { std } \\
58.2\end{array}$ & $\begin{array}{l}\max . \\
463.7\end{array}$ \\
\hline & $\in \mathcal{P}$ & 55.6 & 28.0 & 97.0 & $\in \mathcal{P}$ & 55.2 & 27.8 & 97.0 & $\in \mathcal{P}$ & 59.0 & 27.8 & 97.0 \\
\hline & $\notin \mathcal{P}$ & 36.8 & 44.6 & 344.0 & $\notin \mathcal{P}$ & 32.7 & 33.6 & 313.4 & $\notin \mathcal{P}$ & 51.5 & 59.4 & 463.7 \\
\hline \multirow{4}{*}{$\begin{array}{l}\text { Perc. } \\
\text { dev. }\end{array}$} & & mean & std & max. & & mean & std & max. & & mean & std & max. \\
\hline & all & 0.81 & 1.72 & 9.97 & all & 0.87 & 1.95 & 45.84 & all & 1.04 & 1.91 & 9.97 \\
\hline & $\in \mathcal{P}$ & 6.20 & 2.17 & 9.97 & $\in \mathcal{P}$ & 6.18 & 2.19 & 9.97 & $\in \mathcal{P}$ & 6.65 & 2.37 & 9.97 \\
\hline & $\notin \mathcal{P}$ & 0.49 & 1.02 & 8.28 & $\notin \mathcal{P}$ & 0.56 & 1.42 & 45.84 & $\notin \mathcal{P}$ & 0.71 & 1.25 & 8.28 \\
\hline \multirow{3}{*}{ 2-norm } & all & & 2609 . & & all & & 2149.3 & & all & & 3504. & \\
\hline & $\in \mathcal{P}$ & & 658. & & $\in \mathcal{P}$ & & 654.1 & & $\in \mathcal{P}$ & & 689.3 & \\
\hline & $\notin \mathcal{P}$ & & 2525 . & & $\notin \mathcal{P}$ & & 2047. & & $\notin \mathcal{P}$ & & 3436. & \\
\hline
\end{tabular}


Table 3. (Continued).

Results for the hier16 instance

\begin{tabular}{|c|c|c|c|c|c|c|c|c|c|c|c|c|}
\hline \multirow[b]{2}{*}{ CPU } & \multicolumn{4}{|c|}{$L_{1}$} & \multicolumn{4}{|c|}{$L_{2}$} & \multicolumn{4}{|c|}{$L_{\infty}$} \\
\hline & \multicolumn{2}{|c|}{$\begin{array}{c}\text { Simplex } \\
19.85\end{array}$} & \multicolumn{2}{|c|}{$\begin{array}{c}\text { Int. Point } \\
28.36\end{array}$} & \multicolumn{4}{|c|}{$\begin{array}{c}\text { Int. Point } \\
17.19 \\
\end{array}$} & \multicolumn{2}{|c|}{$\begin{array}{c}\text { Simplex } \\
66.52\end{array}$} & \multicolumn{2}{|c|}{$\begin{array}{c}\text { Int. Point } \\
136.86\end{array}$} \\
\hline \multirow{4}{*}{$\begin{array}{l}\text { Abs. } \\
\text { dev. }\end{array}$} & & mean & std & max. & & mean & std & max. & & mean & std & max. \\
\hline & all & 35.8 & 40.0 & 28 & all & 33.4 & 30.6 & & all & 36.8 & 36.6 & 300.9 \\
\hline & $\in \mathcal{P}$ & 48.3 & 27.4 & 131.0 & $\in \mathcal{P}$ & 48.3 & 27.4 & 131.0 & $\in \mathcal{P}$ & 48.7 & 27.4 & 131.0 \\
\hline & $\notin \mathcal{P}$ & 34.9 & 40.6 & 280.5 & $\notin \mathcal{P}$ & 32.4 & 30.6 & 258.3 & $\notin \mathcal{P}$ & 36.0 & 37.0 & 300.9 \\
\hline \multirow{4}{*}{$\begin{array}{l}\text { Perc. } \\
\text { dev. }\end{array}$} & & mean & std & max. & & $\overline{\text { mean }}$ & std & & & mean & std & $\mathrm{ma}$ \\
\hline & all & 3 & 1.84 & 10.00 & all & 0.90 & 1.81 & & all & 1.13 & 2.05 & 10.00 \\
\hline & $\in \mathcal{P}$ & 89 & 2.38 & 10.00 & $\in \mathcal{P}$ & 6.89 & 2.38 & 10 & $\in \mathcal{P}$ & 7.04 & 2.41 & 10.00 \\
\hline & $\notin \mathcal{P}$ & 43 & 0.78 & 7.59 & $\notin \mathcal{P}$ & 0.50 & 0.75 & 7.59 & $\notin \mathcal{P}$ & 0.73 & 1.26 & 7.59 \\
\hline \multirow{3}{*}{ norm } & \begin{tabular}{|l|} 
all \\
\end{tabular} & & 3203 & & all & & 2706.3 & & all & & 3098.4 & \\
\hline & $\in \mathcal{P}$ & & 830 & & $\in \mathcal{P}$ & & 830.2 & & $\in \mathcal{P}$ & & 836.8 & \\
\hline & $\notin \mathcal{P}$ & & 3094 & & $\notin \mathcal{P}$ & & 2575.9 & & $\notin \mathcal{P}$ & & 2983.2 & \\
\hline
\end{tabular}

Results for the nine12 instance

\begin{tabular}{|c|c|c|c|c|c|c|c|c|c|c|c|c|}
\hline \multirow{5}{*}{$\begin{array}{l}\text { CPU } \\
\text { Abs. } \\
\text { dev. }\end{array}$} & \multicolumn{2}{|c|}{$\begin{array}{c}\text { Simplex } \\
382.13\end{array}$} & \multicolumn{2}{|c|}{$\begin{array}{c}\text { Int. Point } \\
47.38\end{array}$} & \multicolumn{4}{|c|}{$\begin{array}{c}\text { Int. Point } \\
18.29 \\
\end{array}$} & \multicolumn{2}{|c|}{$\begin{array}{c}\text { Simplex } \\
727.28\end{array}$} & \multicolumn{2}{|c|}{$\begin{array}{c}\text { Int. Point } \\
338.8\end{array}$} \\
\hline & & mean & std & max. & & mean & std & max. & & mean & std & max. \\
\hline & all & 36.3 & 44.3 & 490.9 & all & 34.6 & 33.0 & 377.4 & all & 32.6 & 36.5 & 268.0 \\
\hline & $\in \mathcal{P}$ & 51.7 & 28.3 & 154.0 & $\in \mathcal{P}$ & 51.6 & 28.2 & 154.0 & $\in \mathcal{P}$ & 52.1 & 28.2 & 154.0 \\
\hline & $\notin \mathcal{P}$ & 34.4 & 45.6 & 490.9 & $\notin \mathcal{P}$ & 32.4 & 33.0 & 377.4 & $\notin \mathcal{P}$ & 30.1 & 36.7 & 268.0 \\
\hline \multirow{4}{*}{$\begin{array}{l}\text { Perc. } \\
\text { dev. }\end{array}$} & & mean & std & max. & & mean & std & max. & & mean & std & max. \\
\hline & all & 1.35 & 2.34 & 12.55 & all & 1.53 & 2.32 & 25.43 & all & 1.74 & 2.64 & 10.00 \\
\hline & $\in \mathcal{P}$ & 6.71 & 2.38 & 10.00 & $\in \mathcal{P}$ & 6.70 & 2.39 & 11.97 & $\in \mathcal{P}$ & 6.82 & 2.40 & 10.00 \\
\hline & $\notin \mathcal{P}$ & 0.67 & 1.15 & 12.55 & $\notin \mathcal{P}$ & 0.87 & 1.23 & 25.43 & $\notin \mathcal{P}$ & 1.09 & 1.85 & 8.95 \\
\hline \multirow{3}{*}{ 2-norm } & all & & 5840 & & all & & 4878.1 & & all & & 4988.1 & \\
\hline & $\in \mathcal{P}$ & & 2022 & & $\in \mathcal{P}$ & & 2017.2 & & $\in \mathcal{P}$ & & 2034.2 & \\
\hline & $\notin \mathcal{P}$ & & 5478 & & $\notin \mathcal{P}$ & & 4441.5 & & $\notin \mathcal{P}$ & & 4554.5 & \\
\hline
\end{tabular}

Results for the nine5d instance

\begin{tabular}{|c|c|c|c|c|c|c|c|c|c|c|c|c|}
\hline \multirow{4}{*}{$\begin{array}{l}\text { CPU } \\
\text { Abs. } \\
\text { dev. }\end{array}$} & \multicolumn{2}{|c|}{$\begin{array}{c}\text { Simplex } \\
126.67 \\
\end{array}$} & \multicolumn{2}{|c|}{$\begin{array}{c}\text { Int. Point } \\
43.03\end{array}$} & \multicolumn{4}{|c|}{$\begin{array}{c}\text { Int. Point } \\
20.36 \\
\end{array}$} & \multicolumn{2}{|c|}{$\begin{array}{c}\text { Simplex } \\
784.52 \\
\end{array}$} & \multicolumn{2}{|c|}{$\begin{array}{c}\text { Int. Point } \\
137.33\end{array}$} \\
\hline & & mean & std & $\max$ & & mean & std & & & mean & std & max. \\
\hline & all & 41.4 & 68.8 & 1010.0 & all & 37.2 & 37.5 & 499.4 & all & 34.4 & 38.4 & 306.5 \\
\hline & $\in \mathcal{P}$ & 50.6 & 29.3 & 156.0 & $\in \mathcal{P}$ & 50.6 & 29.3 & 156.0 & $\in \mathcal{P}$ & 50.8 & 29.3 & 156.0 \\
\hline \multirow{6}{*}{$\begin{array}{l}\text { Perc. } \\
\text { dev. }\end{array}$} & $\notin \mathcal{P}$ & 39.7 & 73.6 & 1010.0 & $\notin \mathcal{P}$ & 34.7 & 38.3 & 499.4 & $\notin \mathcal{P}$ & 31.4 & 39.1 & 306.5 \\
\hline & & mean & std & $\max$ & & mean & std & $\max$ & & mean & std & max. \\
\hline & all & 1.67 & 2.69 & 10.00 & all & 1.90 & 2.53 & 10.00 & all & 2.23 & 3.02 & 10.00 \\
\hline & $\in \mathcal{P}$ & 6.83 & 2.42 & 10.00 & $\in \mathcal{P}$ & 6.83 & 2.42 & 10.00 & $\in \mathcal{P}$ & 6.87 & 2.42 & 10.00 \\
\hline & $\notin \mathcal{P}$ & 0.73 & 1.31 & 9.78 & $\notin \mathcal{P}$ & 1.00 & 1.11 & 9.31 & $\notin \mathcal{P}$ & 1.38 & 2.25 & 8.79 \\
\hline & \begin{tabular}{|l} 
all \\
\end{tabular} & & 8316 & & all & & 5468.3 & & all & & 5343.4 & \\
\hline \multirow[t]{2}{*}{ 2-norm } & $\in \mathcal{P}$ & & 2383 & & $\in \mathcal{P}$ & & 2383.2 & & $\in \mathcal{P}$ & & 2389.6 & \\
\hline & $\notin \mathcal{P}$ & & 7967 & & $\notin \mathcal{P}$ & & 4921.7 & & $\notin \mathcal{P}$ & & 4779.4 & \\
\hline
\end{tabular}


Table 3. (Continued).

Results for the ninenew instance

\begin{tabular}{|c|c|c|c|c|c|c|c|c|c|c|c|c|}
\hline \multirow{4}{*}{$\begin{array}{l}\text { CPU } \\
\text { Abs. } \\
\text { dev. }\end{array}$} & \multicolumn{2}{|c|}{$\begin{array}{c}\text { Simplex } \\
27.08\end{array}$} & \multicolumn{2}{|c|}{$\begin{array}{c}\text { Int. Point } \\
24.02\end{array}$} & \multicolumn{4}{|c|}{$\begin{array}{c}\text { Int. Point } \\
11.15 \\
\end{array}$} & \multicolumn{2}{|c|}{$\begin{array}{c}\text { Simplex } \\
199.39\end{array}$} & \multicolumn{2}{|c|}{$\begin{array}{c}\text { Int. Point } \\
120.52\end{array}$} \\
\hline & & mean & & & & nean & & & & mean & std & $\max$. \\
\hline & & 41.6 & 0 & 7 & & & & & lall & U & 3.2 & 1020 \\
\hline & D & 52.4 & 28.6 & 192.0 & $\in \mathcal{P}$ & 52.4 & 28.3 & 192.0 & $\in \mathcal{P}$ & 53.0 & 28.3 & 192.0 \\
\hline & $\notin \mathcal{P}$ & 39.9 & 55.5 & 602.7 & $\notin \mathcal{P}$ & 36.6 & 40.0 & 522.8 & $\notin \mathcal{P}$ & 36.8 & 44.7 & 439.1 \\
\hline & & mean & std & $\max$ & & mean & std & $\max$. & & mean & std & $\max$ \\
\hline & all & 1.56 & 2.47 & 16.16 & all & 1.76 & 2.44 & 22.86 & all & 2.19 & 2.93 & 10.00 \\
\hline & $\in \mathcal{P}$ & 6.66 & 2.38 & 10.00 & $\in \mathcal{P}$ & 6.66 & 2.36 & 10.00 & $\in \mathcal{P}$ & 6.79 & 2.39 & 10.00 \\
\hline & $\notin \mathcal{P}$ & 0.79 & 1.29 & 16.16 & $\notin \mathcal{P}$ & 1.02 & 1.35 & 22.86 & $\notin \mathcal{P}$ & 1.50 & 2.32 & 9.93 \\
\hline & $\overline{\text { all }}$ & & $\overline{5447}$ & & all & & $\overline{4444}$ & & all & & $\overline{4708.1}$ & \\
\hline & $\in \mathcal{P}$ & & 1749. & & $\in \mathcal{P}$ & & 1744. & & $\in \mathcal{P}$ & & 1759.5 & \\
\hline & $d \mathcal{D}$ & & 5158. & & $\notin \mathcal{P}$ & & 4087. & & $\notin \mathcal{P}$ & & 4366.9 & \\
\hline
\end{tabular}

Results for the two5in6 instance

\begin{tabular}{|c|c|c|c|c|c|c|c|c|c|c|c|c|}
\hline \multirow{4}{*}{$\begin{array}{l}\text { CPU } \\
\text { Abs. } \\
\text { dev. }\end{array}$} & \multicolumn{2}{|c|}{$\begin{array}{c}\text { Simplex } \\
13.58 \\
\end{array}$} & \multicolumn{2}{|c|}{$\begin{array}{c}\text { Int. Point } \\
16.88\end{array}$} & \multicolumn{4}{|c|}{$\begin{array}{l}\text { Int. Point } \\
9 \\
\end{array}$} & \multicolumn{2}{|c|}{$\begin{array}{c}\text { Simplex } \\
83.48\end{array}$} & \multicolumn{2}{|c|}{$\begin{array}{c}\text { Int. Point } \\
86.47\end{array}$} \\
\hline & & mean & std & $\max$. & & mean & std & $\max$. & & mean & std & max. \\
\hline & all & 38.3 & 52.8 & 530.0 & all & 35.4 & 34.9 & 340.1 & all & 38.3 & 39.3 & 281.8 \\
\hline & $\in \mathcal{P}$ & 49.1 & 32.0 & 169.0 & $\in \mathcal{P}$ & 49.1 & 32.0 & 169.0 & $\in \mathcal{P}$ & 49.7 & 31.8 & 169.0 \\
\hline & $\notin \mathcal{P}$ & 36.7 & 55.0 & 530.0 & $\notin \mathcal{P}$ & 33.5 & 34.9 & 340.1 & $\notin \mathcal{P}$ & 36.7 & 40.0 & 281.8 \\
\hline \multirow{4}{*}{$\begin{array}{l}\text { Perc. } \\
\text { dev. }\end{array}$} & & mean & std & $\max$. & & mean & std & max. & & mean & std & max. \\
\hline & all & 1.46 & 2.49 & 10.00 & all & 1.65 & 2.40 & 17.88 & all & 2.08 & 2.81 & 10.00 \\
\hline & $\in \mathcal{P}$ & 6.80 & 2.42 & 10.00 & $\in \mathcal{P}$ & 6.80 & 2.42 & 10.00 & $\in \mathcal{P}$ & 6.99 & 2.42 & 10.00 \\
\hline & $\notin \mathcal{P}$ & 0.69 & 1.23 & 9.69 & $\notin \mathcal{P}$ & 0.90 & 1.17 & 17.88 & $\notin \mathcal{P}$ & 1.37 & 2.04 & 8.44 \\
\hline \multirow{3}{*}{ 2-norm } & $\overline{\text { all }}$ & & 4917 & & all & & 3749.3 & & all & & 4137.1 & \\
\hline & $\in \mathcal{P}$ & & 1573 & & $\in \mathcal{P}$ & & 1572.0 & & $\in \mathcal{P}$ & & 1582. & \\
\hline & $\notin \mathcal{P}$ & & 4658 & & $\notin \mathcal{P}$ & & 3403.8 & & $\notin \mathcal{P}$ & & 3822. & \\
\hline
\end{tabular}

